\title{
EAl Endorsed Transactions

\section{Effort-based Least Cost Path Link State Scheme for Wireless Body Area Networks using MOD-LEACH Protocol}

\author{
A. Angel Cerli ${ }^{1, *}$, K. Kalaiselvi ${ }^{2}$ \\ ${ }^{1}$ Research Scholar, Department of Computer Science, Vels Institute of Science, Technology\& Advanced Studies(VISTAS), \\ Tamil Nadu (State), India \\ ${ }^{2}$ Associate Professor and Head, Department of Computer Science, Vels Institute of Science, Technology \& Advanced \\ Studies(VISTAS), Tamil Nadu (State), India \\ E mails: ${ }^{1}$ angelcerli@gmail.com, ${ }^{2}$ kalairaghu.scs@,velsuniv.ac.in
}

\section{Abstract}

WBAN (Wireless Body Area Network) is one of the prominent network models which can be either wearable or can be
operated remotely. WSN (Wireless Sensor Network) are combined to form architecture in WBAN. WSN is made up of
multiple sensors that are evenly dispersed. The lifetime of the sensor node is determined by energy efficiency which is the
main design criticality. To receive data that are from Member Nodes (MNs), transmitters of Cluster Head (CH) must always
be kept in on position and Base Station (BS) loses energy, which is due to tasks between clusters. Besides this loss, BS
positioned near CHs is liable to lose additional energy owing to the above process which creates energy inconsistencies.
These issues are overcome by utilizing the proposed work with Effort-based Least Cost Path Link State Scheme (ELCPLS)
calculation with the vitality productive convention Modified Low Energy Adaptive Clustering Hierarchy (MOD-LEACH).
The simulation results are analyzed through parameters like the network throughput, end to end delay, and the residual
energy.

Keywords: Energy efficiency, Residual energy, Routing procedure, Wireless Body Area Network (WBAN).

Received on 16 May 2020, accepted on 03 June 2020, published on 12 June 2020

Copyright (C) 2020 A. Angel Cerli et al., licensed to EAI. This is an open access article distributed under the terms of the Creative Commons Attribution license, which permits unlimited use, distribution and reproduction in any medium so long as the original work is properly cited.

doi: 10.4108/eai.13-7-2018.165280

*CorrespondingAuthor: Email: angelcerli@gmail.com

\section{Introduction}

Various sensors are used in medical healthcare fields which can be wearable. They are also exclusive to monitor and register environmental conditions. WBAN can be fixed inside the human body or can be carried out. They also register the physical conditions of it and organize the data that are gathered at a core location. A message is sent to all the nodes on a network, so that each node in the network remembers for every one of its neighbours, the sequence number of the last link-state message which it received from that node. When a link-state advertisement is received at a node, the node looks up the sequence number it has stored for the source of that link-state message. In [1], defines the importance of monitoring signals of physiological in the human body. However, WBAN's

lifespan is greatly restricted by the life of the battery, and replacing the sensor batteries is not suitable or viable. This battery restriction of WBANs can be potentially the technology of energy-harvesting which is recently developed.

In [2], describes that since consumption of energy is to be taken into consideration, reliability, delay, and characteristics of application are crucial. But, because of the restriction in loss of large propagation and channel's complex conditions, the above-described problems cannot be completely addressed by regular algorithms that exist. When the outbound communication traffic path is selected with the base of cost, loss of propagation takes place.

Every sink or node is capable of transmitting the message to another sink or node clearly stated [5]. Control of Radio power is in every sensor node; based on the distance of transmission, the magnitude can be tuned by the node. The least-cost-path algorithm was discovered to the 
greatest path provided topography, linear characteristics of the beginning and finish points which are referred to as road or canal, other functions about distance, slope, and cost [4][3]. In [6], as each packet of a node is transmitted and every packet of the node is received, some amount of energy is lost by a node. Due to this, the initial energy value in the node is reduced. Energy currently presents in node after packets are either received or transmitted is called residual energy.

The Cluster Heads which lies near BS should forward many packets of data that are originally from the longdistance Cluster Head. Owing to this $\mathrm{CH}$ which is located near BS loses a significant amount of energy along with energy lost intra-cluster task. Problems of Hot spots are caused by the early or premature death of a node. It happened due to unevenness in the consumption of energy. Unequal clustering algorithms can be used to handle this problem. An unequal clustering algorithm is recommended to maintain the balance in the consumption of energy that occurred owing to more inter-cluster traffic. It is also proposed following unequal cluster size. Section 2 explains the literature survey. Section 3 describes the energy Balancing algorithm for the ELCP which is Export LowCost Path Algorithm, and section 4 states the architecture of the recommended project. Section 5 infers analysis of which is followed by a reference.

\section{Related Works}

In this paper, there are flaws in classification as the degree of access priority to the unlicensed spectrum and its coexistence within another wireless system of communication are not considered. EMI which refers to electromagnetic interference, are devices of biomedical which are sensitive. Other hospital machines like infusion pump anaesthesia and incubators do not essentially consist of a system of wireless transmission, but EMI can impact these machines negatively. A recommended method is that which emphasizes the benefits of DLA to identify the least nodes in numbers to protect the requirements of preferred QoS. In the process of selecting a path, many restrictions including reliability which are end-to-end and also delay are considered.

A recommended algorithm has simulated the results and the solutions were verified on its effectiveness. The suggested executing optimization of cross-layer which is between network layers and physical layers (PHY). This is implemented to radio measurement dataset 1 which is experimental. These dataset 1 are being recorded from daily mixed action's and measurement scenarios range in radios which are worn by people. If a node or BAN hub cannot send messages openly to the preferred target node or hub, nearby BAN (Body Area Networks) hubs will be used to relay the message. While node acts as a relay, the node obeys decoding and also sends ahead scheme of relaying which the signal is decoded and later it retransforms it [79].
The authors in [10] deal with the algorithm originally increase the longevity of life of network with minimizing consumption of energy in node with merely sensible output and the ratio of packet delivery. Whereas improvement in Quality of Service (QoS) gives preference to network output enhancement and success rate for WBAN applications packet delivery.

Reference [11] deals with an effective and protected framework that is authenticated, anonymous and contains preservation of location privacy of WBANs which is IoTbased. In a WBAN system, to eliminate malicious user communication, an authentication scheme that is anonymous and efficient in terms of computation is based on the pairing of bilinear. The doctors can efficiently be authenticated by patients without having to know of information about their identity and credentials in this kind of anonymous authentication. As per the literature study, the distance of communication of inter-cluster is determined by elected $\mathrm{CH}$ in cluster similarly distance of communication of intra-cluster is identified by distance among clusters. It also determines the selection of a smart $\mathrm{CH}$ scheme for algorithms of clustering in networks of wireless sensors.

\section{Cluster Head Selection Processes}

Low Energy Adaptive Clustering Hierarchy referred to as $\mathrm{LEACH}$ is the first routing hierarchical protocol. It recommended the fusion of data and also it is a significant breakthrough in protocols of clustering routing. Application of protocol of LEACH is a TDMA that refers to Time Division Multiple accesses that are based on MAC protocol which is known as Media Access Control Protocol. The primary objective of the protocol is to enhance networks of wireless sensor's lifetime. It is achieved by reducing consumption of energy which is needed $\mathrm{CH}$ creation and maintenance.

To periodic data collection, LEACH with cluster-based is generally recommended for applications of WSNs. The protocol makes use of the probabilistic methodology to select the node as a $\mathrm{CH}$ and transfers cumulated data to $\mathrm{BS}$ with the help of the communication of single-hop. To maintain balance in the consumption of energy, $\mathrm{CH}$ 's role is rotated within other nodes frequently. When using a cooperative communication method, a greater number of nodes which are called cooperative nodes is involved in the transmission of the same data to target with source node. The transmission's total energy requirement is minimized compared to other methods [12]. Persistence of lifetime of network and transmission of energy-efficient determines the network's lifetime. Batteries are used to power nodes in nearly all applications. Energy should be used to a minimum level as the supply of very low energy is with the batteries. The reason is that the node's lifespan is predominantly dependent on the battery's lifetime. The nodes which are located nearby the sink shall die before other nodes of the network. The reason is that nodes near to the sink have heavy data loading from other nodes of the 
network. So, it needs higher energy in processing these data. Because of this, it dies early. Hence, all data will not attain the destination. BN's power utilization is derived for the successful finishing of the transfer of data. The power utilized in the Sleep condition is also considered in the calculation of the work. The time between sending of wakeup packet and receipt of acknowledging packet is defined as the delay and it is also calculated. We also calculated suggested MAC protocol's (Media Access Control) average utilization of power and delay. Average power when in sleep condition, wake up condition, and state of communication of every $\mathrm{BN}$ in a time scale which is fixed in the inter-arrival time of packets are also included here. As the recommended protocol is on-demand in nature, it consumes minimum power [13].

\subsection{Traffic and Energy Heterogeneities in WBAN}

The efficient number of bits concerning communication round is increased by raising heterogeneity of traffic, maximizing the length of packet nodes. This raises the utilization of WSN energy per round and decreases the lifetime of WSN (period of stability).

$$
T\left(i_{t}, r\right)=\left\{\begin{array}{c}
\left.\frac{P i_{t(r)}}{1-P i}{ }_{t(r)\left(r \bmod \frac{1}{P i_{t}(r)}\right)}\right) \text { if node } i \epsilon G \\
0
\end{array}\right.
$$

Where $P i_{t(r)}$ is the $\mathrm{CH}$ selection possibility for the node $i_{\mathrm{t}}$ during the round $r$. G(r) is said to be the suitable set of nodes for round (r). The capability for node $i_{t}$ in rotational approach is $\frac{1}{P_{i_{-} t(r)}}$. The possibility to be $\mathrm{CH}$ of node $\mathrm{I}$ in round $\mathrm{r}$ is explained as

$$
P i_{t(r)}=\frac{P_{o p t . N\left(1+\propto_{e h_{i}}\right) N\left(1+\propto_{t h^{-}}{ }_{e h_{i}}\right) E_{i}(r)}}{\left(N+\sum_{i=1}^{N} \propto_{e h_{i}}\right)\left(N+N_{\propto_{t h}}{ }^{-\propto_{T o}}\right) E_{A v g}(r)}(2)
$$

$E_{A v g}(r)$ is a average energy of the round and the $P_{o p t}=\frac{K_{o p t}}{N}$ be a perfect probability of a node to be $\mathrm{CH}$,

$$
E_{\text {Avg }}(r)=\frac{1}{N} E_{\text {Tot }}\left(1-\frac{r}{R}\right) ; \quad \text { Where } R=\frac{E_{\text {Tot }}}{E_{\text {Round }}}
$$

Here $\mathrm{R}$ is the network life estimated value in terms of the number of rounds based on homogeneous energy dissipation in every round. The energy of the network might not exhaust in homogeneously.

\subsection{Energy Efficient Protocol}

WBANs energy efficiency is specifically based on two methodologies: 1) low power PHY layer design and 2) efficient transmission protocol design. Low power PHY design is identified by transceivers of low-power and design factors of the sensor. Efficient transmission protocol designs consist of proficient protocols of MOD - LEACH and LEACH, proficient packet scheduling, controls of admission, etc. Allocation of the channel can be controlled and coordinated by them [14]. It also assures reliability and reduces the consumption of energy from the utilization of high channel. The protocol processes are compacted and well managed with the environment of a uniform sensor. As per the protocol, new $\mathrm{CH}$ is selected for each round so the new cluster is to be formed. Hence, it results in routing overhead which is unwanted which in turn ends in extreme utilization of energy that is limited. In case $\mathrm{CH}$ did not consume more energy in the proceeding round, then some node with less energy may restore it as $\mathrm{CH}$ in the process of selecting subsequent $\mathrm{CH}$. The current $\mathrm{CH}$ 's residual energy, it is, necessary to control $\mathrm{CH}$ change in each round. So, to save energy, it is needed to have a replacement algorithm of an efficient cluster head. As MOD-LEACH has the scheme of replacement of proficient $\mathrm{CH}$ and power transmission lever which is dual for communication of intra-cluster and inter-cluster, MOD-LEACH's network lifetime has a better constant period. The greatest lifetime of the network is provided by MOD-LEACH with Soft Threshold (MOD-LEACHST) among all other protocols. This is because it has a limitation in several transmissions (soft threshold concept) with a mechanism of replacing $\mathrm{CH}$ which is efficient which saves energy internationally and various levels of power for communication of intra-cluster and inter-cluster.

\subsection{MOD- LEACH}

The primary disparity of protocol of MOD-LEACH comparison to LEACH is the way of selection of $\mathrm{CH}$, where at each recent round, it modifies $\mathrm{CH}$ and it shall not obtain other options to be selected for next $1 / \mathrm{p}$ rounds. "Efficient cluster head replacement scheme" is introduced in Modify LEACH that is a verge informing of $\mathrm{CH}$. As long as $\mathrm{CH}$ does not spend more energy in its duration and is having energy which is more than that of the threshold required, $\mathrm{CH}$ will still be $\mathrm{CH}$ to the subsequent round. Hence, Energy exhausted in packet routing of new $\mathrm{CH}$ and forming clusters is saved by this method. However, when $\mathrm{CH}$ is having energy lesser than that of the threshold, it shall be reinstated [15].

A lifetime of the network's efficiency is improved by energy saving with the help of MOD LEACH. Calculation of an optimal number of $\mathrm{CHs}$, because a greater number of $\mathrm{CHs}$ will observe more amount of energy and reduced network lifetime, according to the position of BS in the field (a $\mathrm{CH}$ should not be very far to $\mathrm{BS}$ ), square root of field area and the total number of sensors nodes as specified by below formula (2).

$$
\mathrm{K}_{\text {opt }}=\sqrt{\frac{N}{2 \pi}} \times \sqrt{\frac{\epsilon f_{S}}{\epsilon_{\text {amp }}}} \times \frac{M}{{\text { dist_to_} B S^{2}}^{2}}
$$

- Percentage of CHs not fixed and recalculated each recent round depending on number of active nodes by the formula given below (5).

$$
\text { Dist to } \mathrm{BS}=\sqrt{\left(\left(X_{b s}-\text { length }\right)^{2}+\left(Y_{b s}-\text { width }\right)^{2}\right)}(4)
$$


- Each node selects which cluster it links in an existing round which is based on residual energy and $\mathrm{CH}$ distance which is specified by the below formula (6).

$$
\begin{aligned}
& \mathrm{M}=\sqrt{\left(\text { Network }_{\text {length }} * \text { Network }_{\text {width }}\right)} \\
& \mathrm{P}_{\mathrm{CN}}=\frac{\mathrm{N}}{\mathrm{k}_{\mathrm{opt}}}(6) \\
& \text { Ratio }\left(E_{\text {res }}, d_{\text {nodeito } H_{j}}\right)=\frac{E_{\text {res }}\left(\mathrm{CH}_{i}\right)}{d_{\text {nodeito } \mathrm{CH}_{j}}}
\end{aligned}
$$

Below figure shows the Setup phase algorithm.

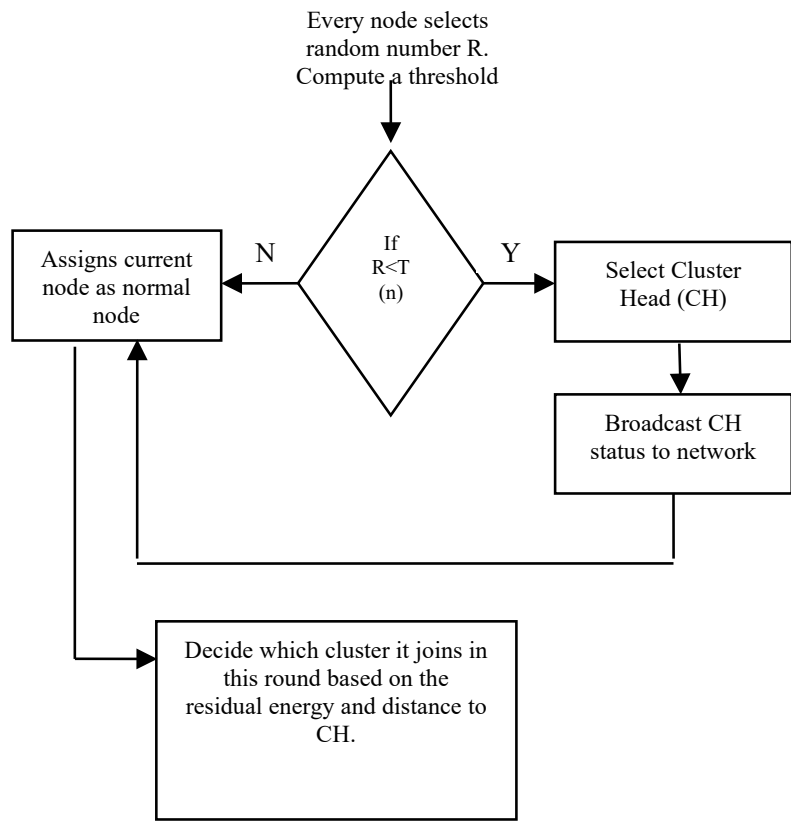

Figure 1. Setup phase Algorithm for MOD- Protocol

When the destination of the lowest-cost path is modified, the update is sent to neighbor's nodes by middle nodes. It is shown in figure 1 . Besides threshold, random number $\mathrm{R}$ is chosen by the phase of setup. The situation with $\mathrm{R}<\mathrm{T}(\mathrm{n})$, shall hold the state with the selected $\mathrm{CH}$ be true provides the broadcast besides network status. Normal of self is selected with the situation of T(n) along with G. Residual energy and $\mathrm{CH}$ distance determines the normal state for the cluster's choice besides joints. The nodes which should originally be aware of the characteristics of other nodes within the network should only be aware of characteristics and costs to its neighbors which are openly associated. It is done by receipt of the broadcast of the link state which is in general from other nodes. The algorithm is explained below.

\subsubsection{Effort-Based Least Cost Path through Link State Algorithm}

Step 1: Start

Step 2: Cluster Organization of N numbers

Step 3: Grouping of Intra-cluster and Inter-cluster

Case I: Intra-cluster distances are the smallest

Step 4: To identify the least-cost path from a source to a destination needs finding a series of links.

- The first link in the path is linked to the source.

- The last link in the path is linked to the destination.

- $\quad$ For all $i$, and $i$ and $i-1 \mathrm{st}$ link in the path are linked to the similar node for the minimum cost path.

- To compute the total of the cost of the links on the path is the least overall potential paths in between destination and the source.

Case II: Inter-cluster distances are greatest.

Step 5: Worse Case Condition: To identify the least cost paths from the source to all destinations

- $\quad$ First Iteration: all $n$ nodes are searched through to define the node $w$, not in $\mathrm{N}$ that has the least cost.

- Second iteration: $n-1$ nodes needed to be checked to identify the least cost.

- $\quad$ Third iteration $n-2$ nodes and so on.

Step 6: Overall, the total number of nodes needed to seek through all the iterations is $n^{*}(n+1) / 2$.

Case III: Link State Checking condition: implementation of the link-state algorithm has the worst-case complexity of order $n$ squared: $O(n 2)$. When the time in linear time and reducing the complexity.

Step 7: End

All these nodes are stable in state and dispersed unsystematically. BS is rigid and situated in the middle of the sensors. All these nodes situated in the network are uniform and start along with having a capacity of equal energy level. Data is located in Nodes for sending and distance from receiver and node determines the power to transmit. The above-said algorithm creates the link which is based on the path of least cost which is based on the effort that linked with a link-state algorithm.

\subsection{System Model for Wireless Body Area Network}


The system model defines feeds provided to WBAN including lactic acid, temperature, ECG, left Arm, glucose, knee, and calf, left and right palm. The system model defines feeds provided to WBAN including lactic acid, temperature, ECG, left Arm, glucose, knee, and calf, left and right palm.

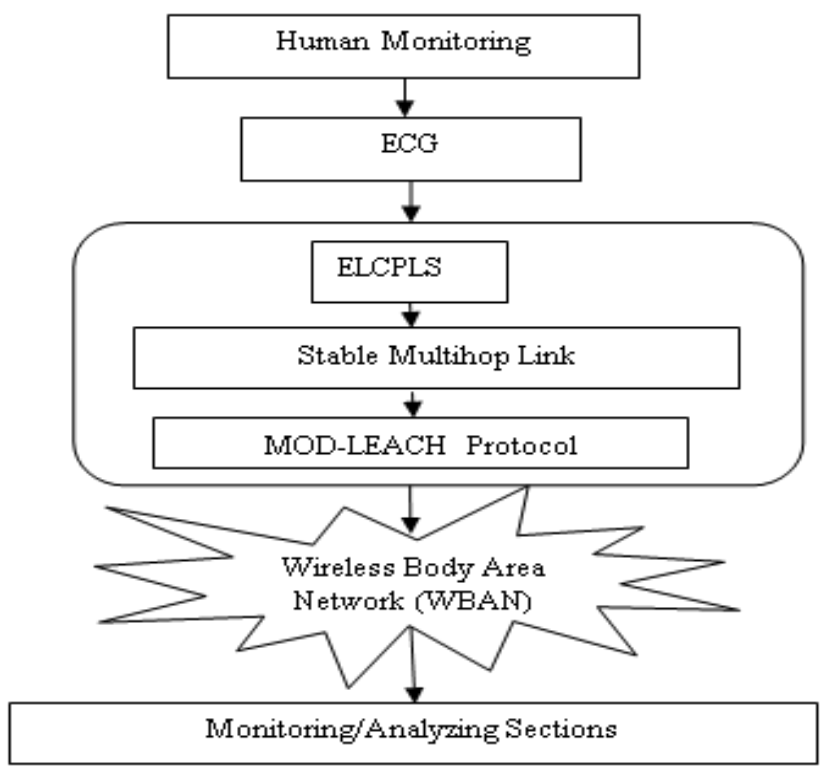

Figure 2. Wireless Body Area Network for effective passing of data

\section{Simulation Result Analysis}

The parameter values are discussed in this section. The values are optimizing by using in the simulation results shown in the below table 1 .

\section{Table 1. Parameter Values}

\begin{tabular}{|c|l|c|}
\hline S.No. & \multicolumn{1}{|c|}{ Parameter } & Value \\
\hline 1. & Area & $(3.5 \mathrm{~m}, 3 \mathrm{~m})$ \\
\hline 2. & Number of nodes & 200 \\
\hline 3. & Initial Energy & $2 \mathrm{~J}$ \\
\hline 4. & Radio range & $70 \mathrm{~cm}$ \\
\hline 5. & Network interface type & $\begin{array}{l}\text { Wireless Physical } \\
\text { layer }\end{array}$ \\
\hline 6. & Energy Model & Energy Model \\
\hline 7. & Tx Power & $0.066 \mathrm{~J}$ \\
\hline 8. & Rx Power & $0.0395 \mathrm{~J}$ \\
\hline 9. & Idle Power & $0.0035 \mathrm{~J}$ \\
\hline 10. & Packet size & $70 \mathrm{bits}$ \\
\hline 11. & MAC & IEEE802.154 \\
\hline 12. & Time & $100 \mathrm{~s}$ \\
\hline
\end{tabular}

MOD- LEACH kind of protocol is utilized in suggested work. A pulse of input ECG which is obtained in process is given in results below and main path and system model fails, ELCPLS which is Effort Low-Cost Path Model through Link State Algorithm shall utilize the substitute path of data transmission to minimize data transmission delay, after that it eliminates local congestion and minimizes delay of the queue.

\subsection{Simulation Results}

ECG plot's input which is for the signal of ECG that is detected in the human of the timing of detecting stress is shown in figure 3. The amplitude of the signal is represented in $\mathrm{Y}$-axis and Timing is plotted in $\mathrm{X}$-axis. The network diagram of $\mathrm{CH}$ WBAN is stated in figure 4 . It is generated with the help of a model of the least-cost path of effort. Nodes that are active and located in $\mathrm{CH}$ are represented in green color and nodes in red color refers to the $\mathrm{CH}$ of intra-cluster head nodes and internodes.Nodes that are not located inactive links are shown as blue color nodes indicated in figure 5. The path of an active link which is done with the help of the algorithm of the link path is shown in red color node surrounded by a yellow circle. The specific link is used to transmit data. Figure 6 states the protocol MOD- LEACH that minimizes high energy consumption giving other details like $\mathrm{X}$-axis referring number of rounds and path loss, nodes which are dead, packets related to Cluster Head, packet to Base Station and also the number of $\mathrm{CH}$. The simulation results are following in below:

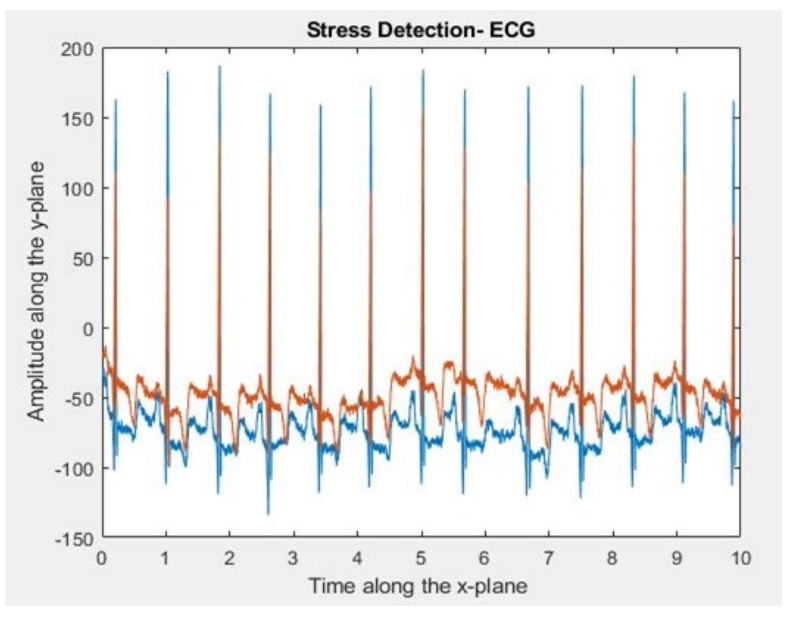

Figure 3. Input ECG signal plot 


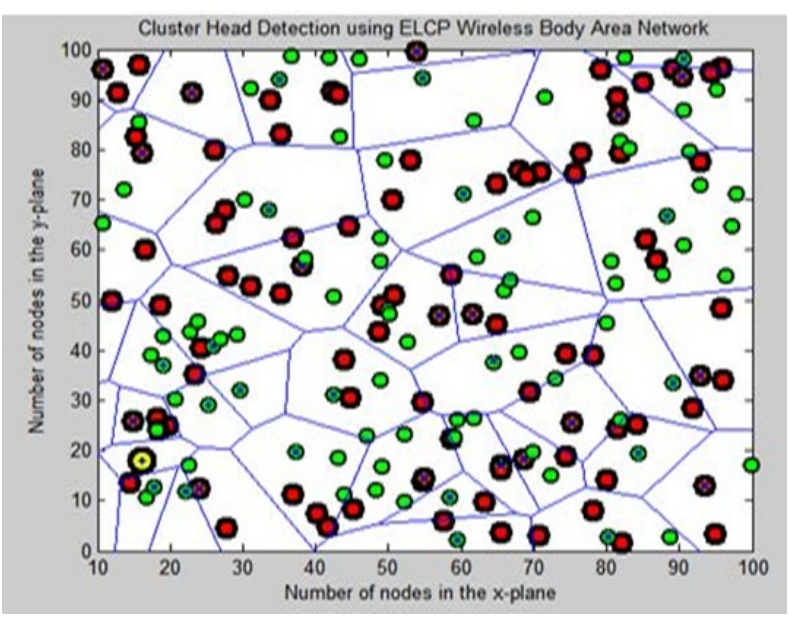

Figure 4. Wireless Body Area Network

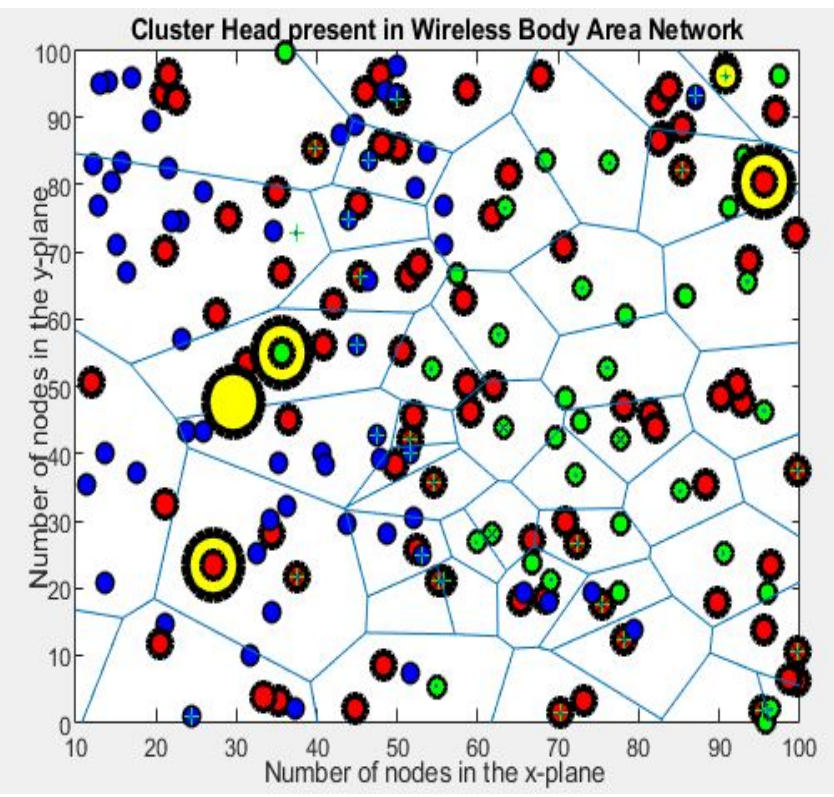

Figure 5. Cluster Head and ELCP_LS
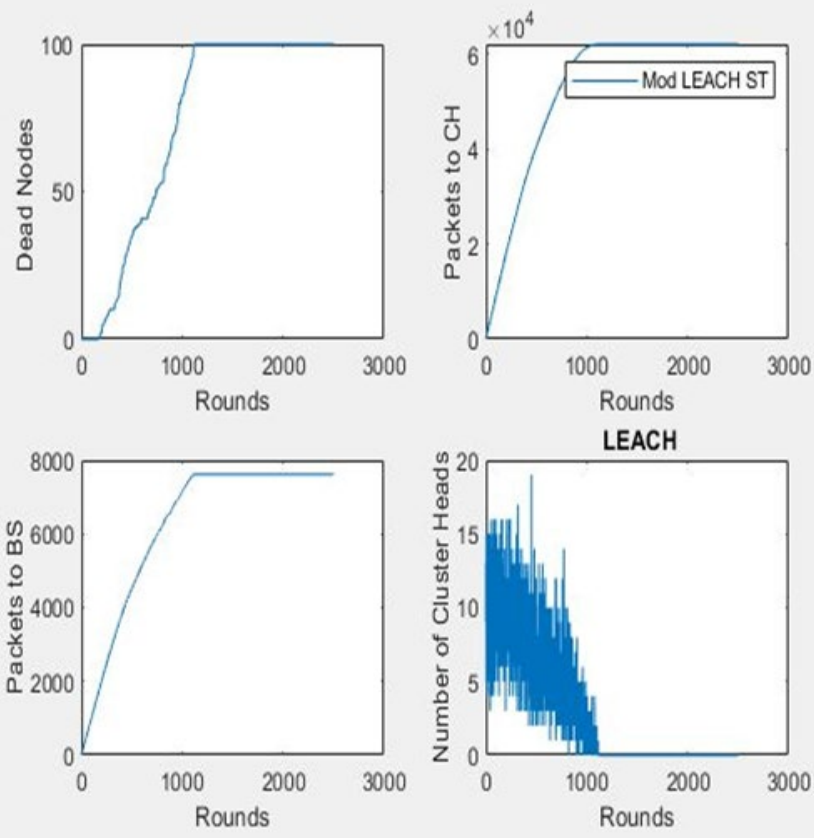

Figure 6. MOD - LEACH protocol

Relation with dead nodes with rounds is given in figure 7 and 8 . In 4 to 5 cluster nodes and until rounds of 2000 nodes of inter-cluster looks constant. Around 3000 and 4000 rounds, nodes of intra-cluster seems stabilized. In 8000 rounds, data packets which are thrown to sink (i.e.) WBAN transferred data packets 70bits transformation happens to the sink node. Drop-in data packets of WBAN are shown in the above figure 9 as it shows data packet drop in intra-cluster looks more than the drop in inter-cluster. In the round of 5000, data packets are gathered in link. The complete transfer of data packet happens in the rounds near 3000 shown in figure 10.

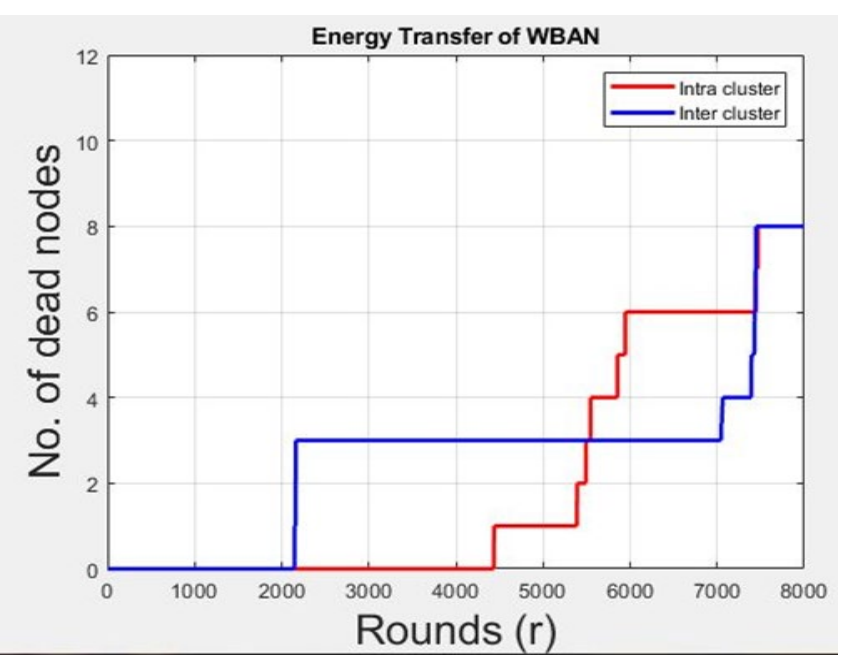

Figure 7. Energy Transfer for WBAN 


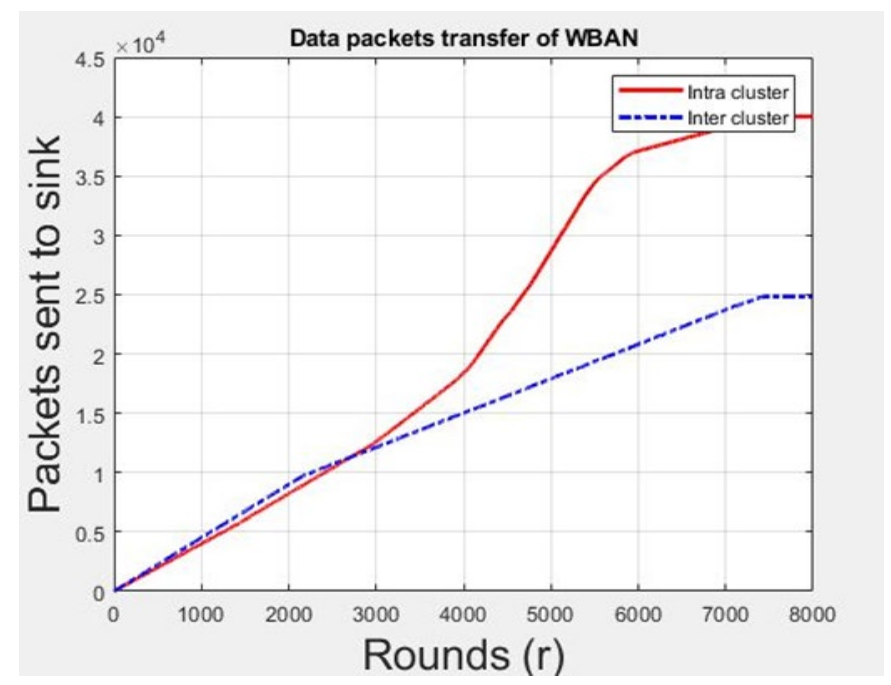

Figure 8. Data packet Transfer for WBAN

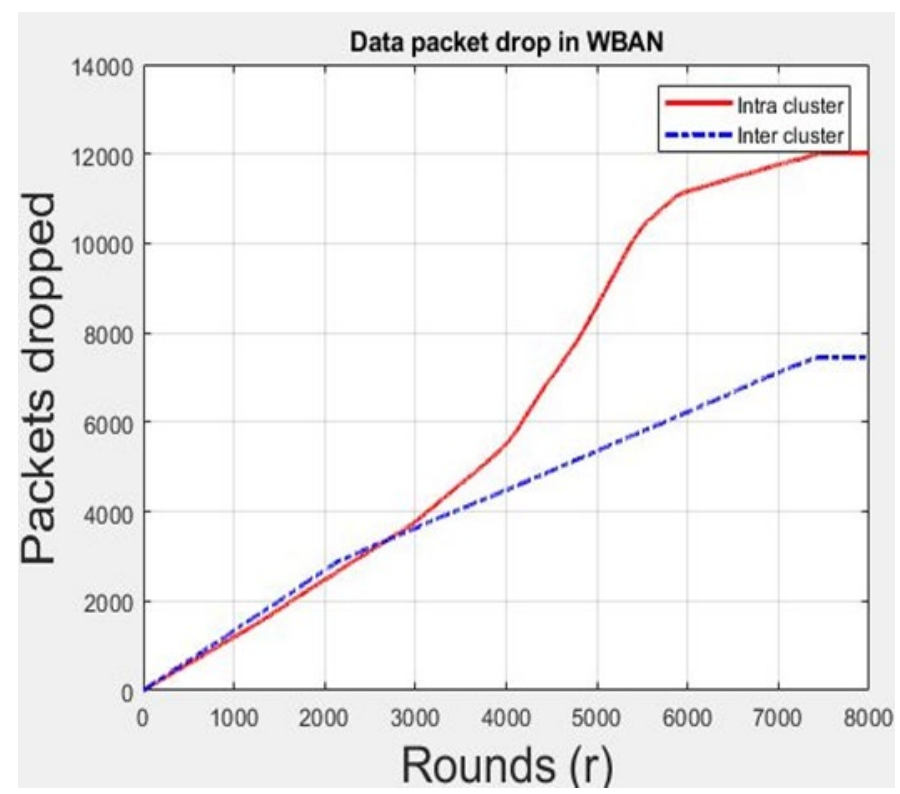

Figure 9. Data packet drop in WBAN

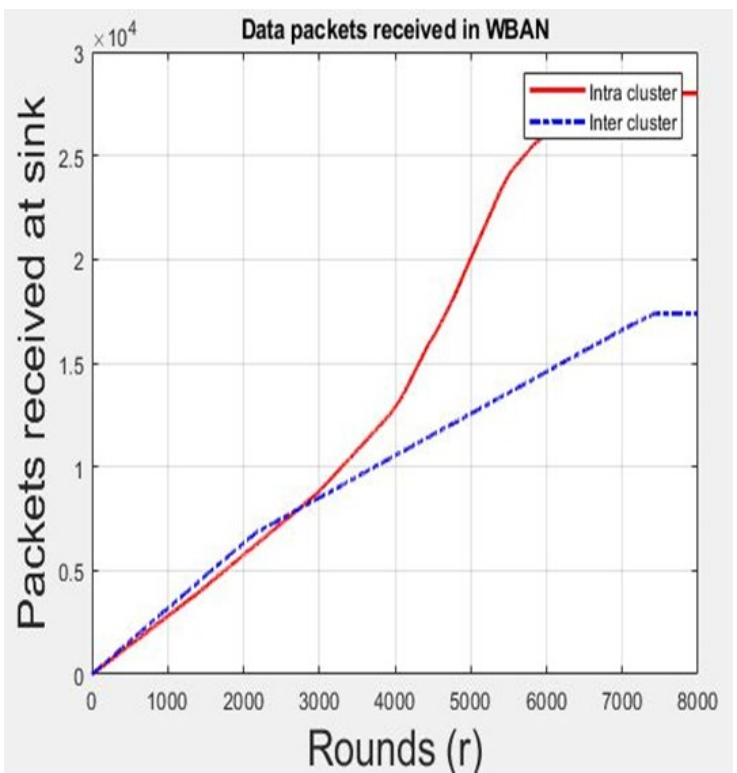

Figure 10. Data packet received in WBAN

In WBAN, residual energy which is received is shown in figure 11. The residual energy $(\mathrm{J})$ in figures 11 mentions that residual energy and near to end as it reaches rounds from 7000 to 8000 . Path loss which is taken place in WBAN is given in figure 12 . At around 7500 , both intercluster and intra-cluster flow against which is path loss direct to end.Figure 13 specifies the delay of propagation of WBAN. Delay takes place here in inter and intra-cluster. Reline mentioned in the graph refers to the distance within nodes located in $\mathrm{CH}$. This region shows the distance is maintained is least. WBAN's delay is shown in figure 14 . Here intra-cluster distance is sustained with equal delay which is nearly 7500 rounds, if the delay is less and standard with ground's length.

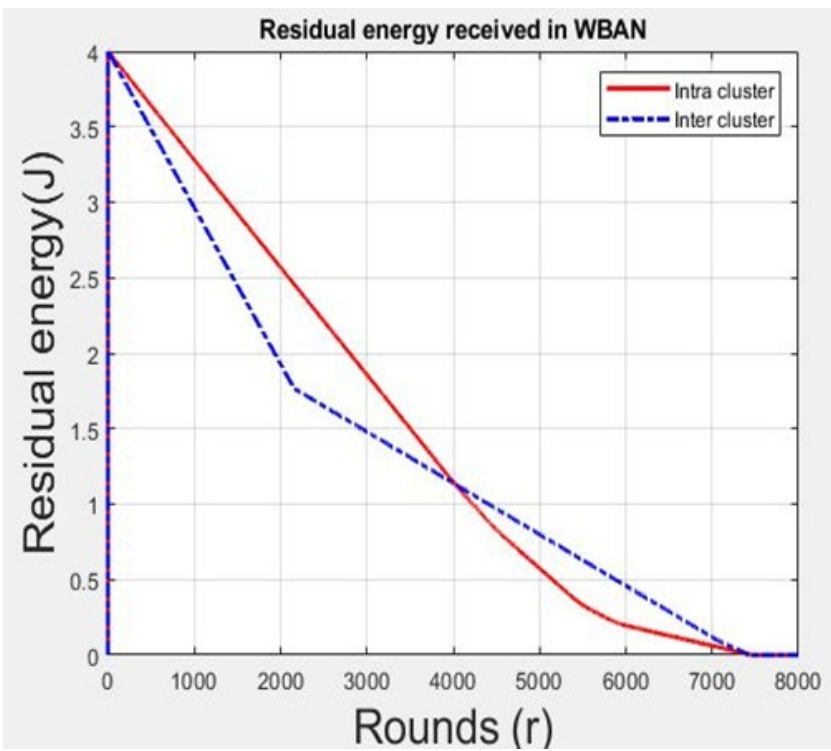

Figure 11. Residual Energy received in WBAN 


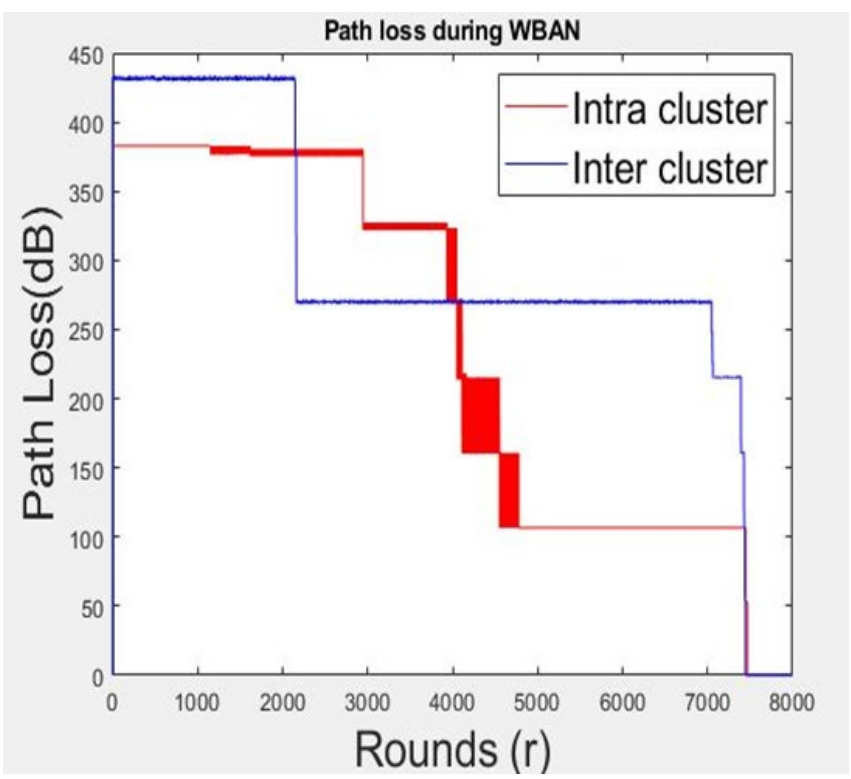

Figure 12. Path loss in WBAN

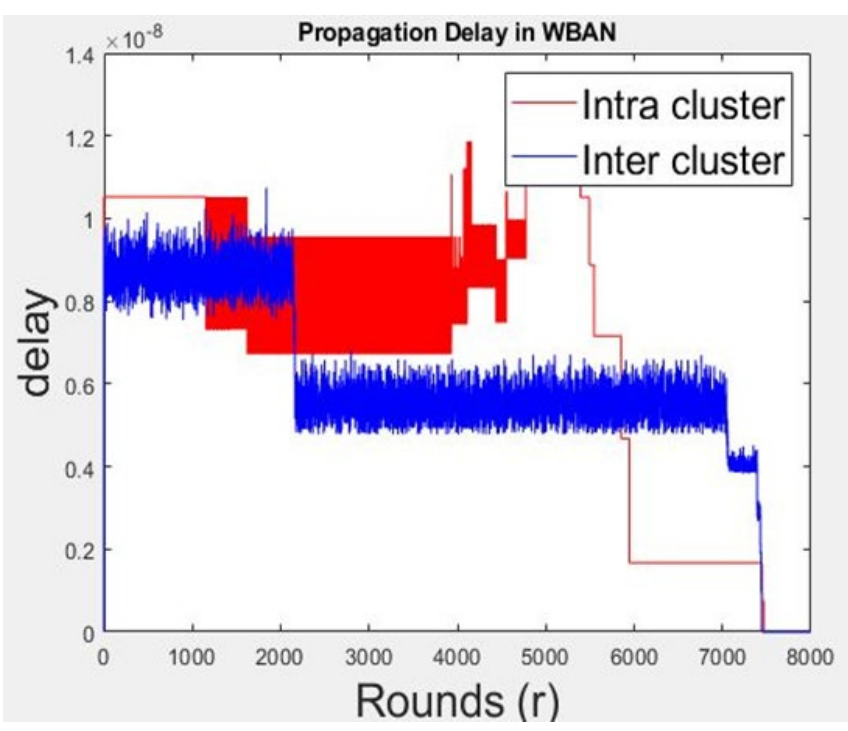

Figure 13. Propagation delay in WBAN

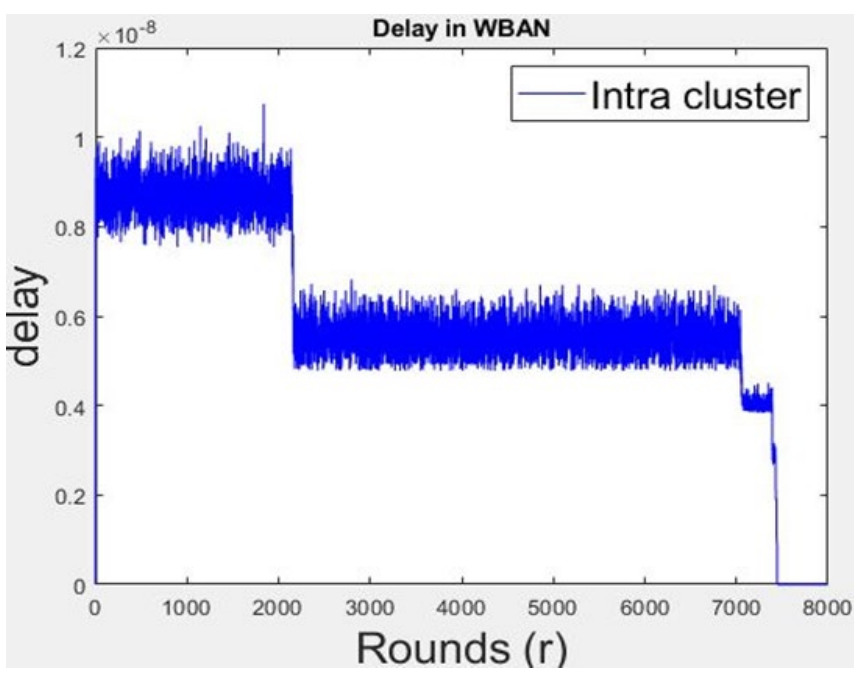

Figure 14. Delay in WBAN

\section{Comparision Graph}

Comparison has been given here the end-to-end delay of WBAN. Three algorithms including Stabilise link protocol, ELCPLS, and SEAR which is meant to Simplified EnergyBalanced Alternative-Aware Routing Algorithm are compared here. Figure 15 states the delay of end to end in WBAN the delay taken place here owing to, routing of long-distance, the traffic, selection of $\mathrm{CH}$ in the processesof network. The recommended work selects the middle cluster along with the least distance between the nodes at the end to end delay.

SEAR and Stablished link algorithm which is the protocol of comparison are in comparison fewer in the domain. Figure 16 states Cluster Heads next to Base Station should send ahead many data packets that are basically from the long distant $\mathrm{CHs}$. Owing to this $\mathrm{CH}$ which is located near BS loses a significant amount of energy along with energy lost intra-cluster task. Problems of Hot spots are caused by the early or premature death of a node. The algorithm of Unequal clustering effectively deals with this problem of the hot spot.

Using $\mathrm{CH}$ formation, the least cost algorithm is based on effort and algorithm of list state which refers to minimum distance as the cost can eliminate these problems.Link within the minimum distance path is provided by the linkstate algorithm. The recommended work continues to have the energy level of $\mathrm{CHs}$ located near to $\mathrm{BS}$, while $\mathrm{CH}$ nodes are occupied in transmission of high data.The throughput of the network for WBAN maximizes owing to the appropriate choice of $\mathrm{CH}$ in addition to the lesser distance along with an appropriate route path. If $\mathrm{CHs}$ distance is shortest, it will minimize traffic of path in the network. The network throughput can be eliminated with the help of the shortest path which is between the $\mathrm{CH}$ node and target node id stated in figure 17.

End to End Delay 


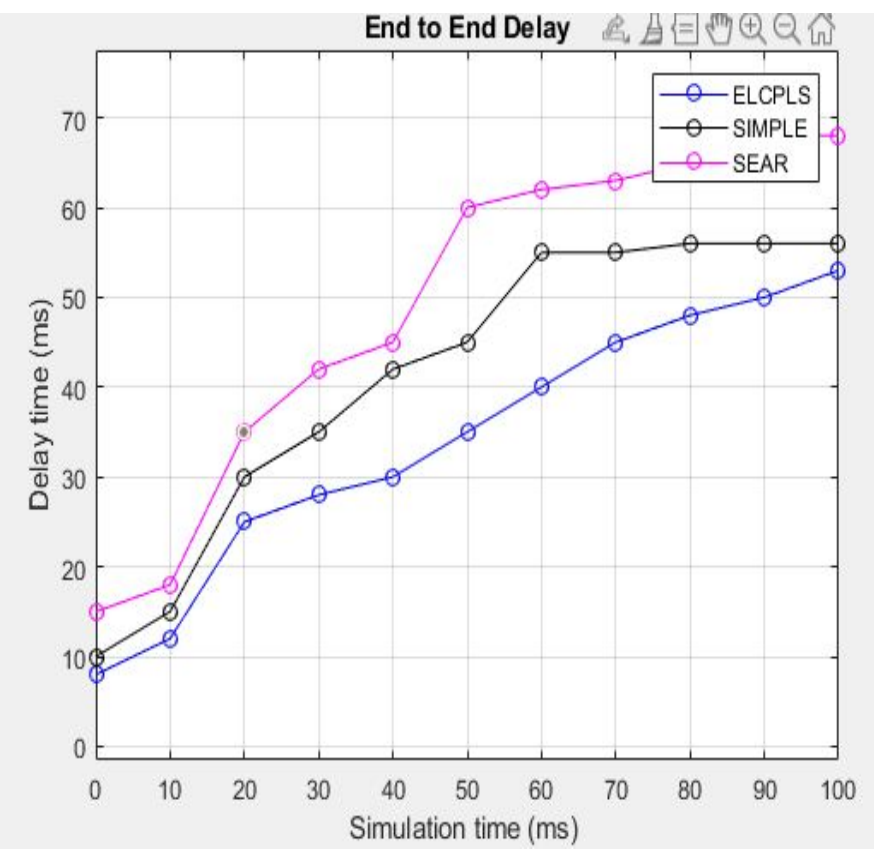

Figure 15. End to End Delay in WBAN

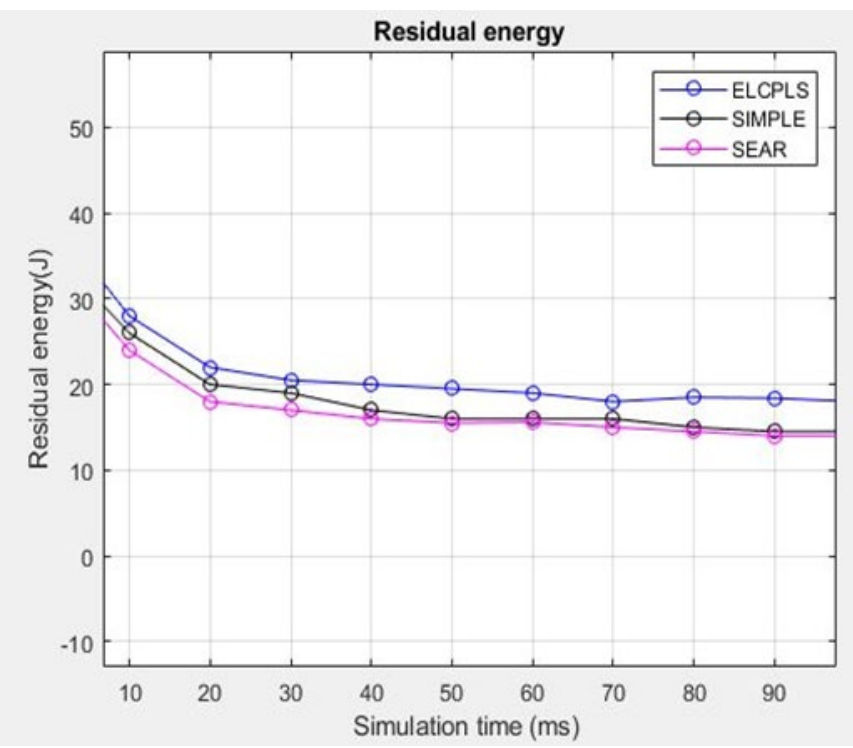

Figure 16. Residual energy in WBAN

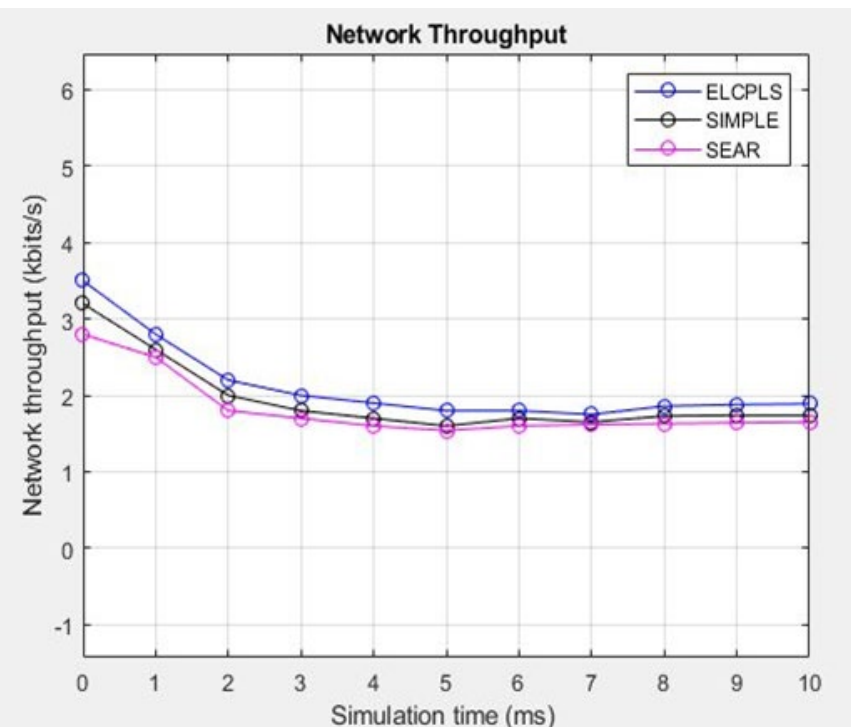

Figure 17. Network throughput in WBAN

\section{Conclusion}

The recommended framework utilizes ECG which is ElectroCardiogram signals which are attained from the human being who appears to be stressful.The framework which is designed will allow pulse signal to pass through. It is based on the low-cost path concept and algorithm of link state are used to connect proper links. To transmit the data with the help of less energy $\mathrm{CH}$ nodes which are located near to target nodes, the protocol of MOD- LEACH and above algorithms are used in designed work. The cluster head Wi-Fi frame space network using the concept that of the effort least value path type for the device approaches. The vitality productivity for the convention is contrasted and the Simplified Energy-Balanced Alternative-Aware Routing Algorithm (SEAR) increments in the remaining vitality give better execution. Here the link-state protocol is performed on every switching node in the network, where every node constructs a map of the connectivity to the network, in the form of a graph, showing the connection of nodes in the network. The collection of these best paths will then form each node's routing table. The energy consumption can be reduced in node to a node during the data transmission. The main novelty of this work is to reduce the energy consumption using MOD-LEACH protocol with the help of Effortbased Least Cost Path Link State Scheme (ELCPLS) in WBAN.Medical applications include real-time, continuous patient monitoring by which pre-hospital, in-hospital, and ambulatory monitoring can be possible thereby helping to replace expensive and cumbersome wired systems. The people suffering from home can easily update the condition of the patient to relatives as well as the physician. The recommended work maximizes the energy of residual close to the target node. The wireless node data transmission with diminishing energy consumption using ZIGBEE is 
used to implement the future work of healthcare applications.

\section{References}

[1] Shuai Shen, "A Sum-Utility Maximization Approach for Fairness Resource Allocation in Wireless Powered Body Area Networks",2019.

[2] Mu, Jiasong, Xiang Liu, and Xiangdong Yi. "Simplified Energy-Balanced Alternative-Aware Routing Algorithm for

[3] Wireless Body Area Networks." IEEE Access 7: 108295$108303,2019$.

[4] Collischonn, Walter, and Jorge Victor Pilar."A direction dependent least-cost-path algorithm for roads and canals" International Journal of Geographical Information Science 14, no. 4: 397-406,2000.

[5] Mahajan, Shilpa, and Jyoteesh Malhotra. "Energy efficient path determination in wireless sensor network using BFS approach" Wireless Sensor Network 3, no. 11: 351, 2011.

[6] Jiang, Xin, et al. "ELCP: An Effort-Based Least Cost Path Scheme for MANETs." 2009 Fifth International Conference on Mobile Ad-hoc and Sensor Networks. IEEE, 2009.

[7] Kim, Sun-Kyum, Jae-Ho Choi, and Sung-Bong Yang. "Hotspot: Location-based Forwarding Scheme in an Opportunistic Network." Adhoc\& Sensor Wireless Networks 26 (2015).

[8] Sodagari, Shabnam, Bahareh Bozorgchami, and Hamid Aghvami. "Technologies and challenges for cognitive radio enabled medical wireless body area networks" IEEE Access 6: 29567-29586, 2018.

[9] Mostafaei, Habib. "Energy-efficient algorithm for reliable routing of wireless sensor networks", IEEE Transactions on Industrial Electronics 66, no. 5567-5575, 2018.

[10] Shimly, Samiya M., David B. Smith, and SamanehMovassaghi. "Experimental Analysis of Crosslayer Optimization for Distributed Wireless Body-to-Body Networks." IEEE Sensors Journal 2019.

[11] Kefa G. Mkongwa, "Link Reliability and Performance Optimization In Wireless Body Area Networks", IEEE Access 2018.

[12] Vijayakumar, P., Mohammad Obaidat, Maria Azees, SkHafizul Islam, and Neeraj Kumar. "Efficient and Secure Anonymous Authentication with Location Privacy for IoTbased WBANs", IEEE Transactions on Industrial Informatics 2019.

[13] Purkar, Santosh V., and Rajkumar S. Deshpande. "Energy efficient clustering protocol to enhance performance of heterogeneous wireless sensor network: EECPEP-HWSN." Journal of Computer Networks and Communications 2018.

[14] Javaid, Nadeem, Mehreen Shah, Ashfaq Ahmad, Muhammad Imran, Majid Khan, and Athanasios Vasilakos. "An enhanced energy balanced data transmission protocol for underwater acoustic sensor networks." Sensors 16, no: 4, 2016.

[15] Huang, Zhirui, Lip Yee, Tan Fong Ang, Mohammad Hossein Anisi, and Mohammed Sani Adam. "Improving the Accuracy Rate of Link Quality Estimation Using Fuzzy Logic in Mobile Wireless Sensor Network." Advances in Fuzzy Systems 2019.

[16] Sangeethapriya, S., and R. Amutha. "Reliable data transmission in wireless sensor networks", In International Conference on Information Communication and Embedded Systems (ICICES2014), pp. 1-6. IEEE, 2014. 